\title{
Altered bile acid profile in mild cognitive impairment and Alzheimer's disease: Relationship to neuroimaging and CSF biomarkers
}

\author{
Kwangsik Nho ${ }^{\mathrm{a}, 1}$, Alexandra Kueider-Paisley ${ }^{\mathrm{b}, 1}$, Siamak MahmoudianDehkordi ${ }^{\mathrm{b}, 1}$, \\ Matthias Arnold ${ }^{\mathrm{b}, \mathrm{c}}$, Shannon L. Risacher ${ }^{\mathrm{a}}$, Gregory Louie ${ }^{\mathrm{b}}$, Colette Blach ${ }^{\mathrm{d}}$, Rebecca Baillie ${ }^{\mathrm{e}}$, \\ Xianlin $\operatorname{Han}^{\mathrm{f}}$, Gabi Kastenmüller ${ }^{\mathrm{c}, \mathrm{g}}$, Wei Jia ${ }^{\mathrm{h}}$, Guoxiang Xie ${ }^{\mathrm{h}}$, Shahzad Ahmad ${ }^{\mathrm{i}}$, \\ Thomas Hankemeier ${ }^{j}$, Cornelia M. van Duijn ${ }^{i}$, John Q. Trojanowski ${ }^{k}$, Leslie M. Shaw ${ }^{k}$, \\ Michael W. Weiner', P. Murali Doraiswamy ${ }^{\mathrm{b}, \mathrm{m}, \mathrm{n}}$, Andrew J. Saykin ${ }^{\mathrm{a}, * *}$, \\ Rima Kaddurah-Daouk $^{\mathrm{b}, \mathrm{m}, \mathrm{n}, *}$, for the Alzheimer's Disease Neuroimaging Initiative and the \\ Alzheimer Disease Metabolomics Consortium ${ }^{2}$ \\ ${ }^{a}$ Department of Radiology and Imaging Sciences, Center for Computational Biology and Bioinformatics, and the Indiana Alzheimer Disease Center, Indiana \\ University School of Medicine, Indianapolis, IN, USA \\ ${ }^{b}$ Department of Psychiatry and Behavioral Sciences, Duke University, Durham, NC, USA \\ ${ }^{c}$ Institute of Bioinformatics and Systems Biology, Helmholtz Zentrum München, German Research Center for Environmental Health, Neuherberg, Germany \\ ${ }^{d}$ Duke Molecular Physiology Institute, Duke University, Durham, NC, USA \\ ${ }^{e}$ Rosa \& Co LLC, San Carlos, CA, USA \\ ${ }^{f}$ University of Texas Health Science Center at San Antonio, San Antonio, TX, USA \\ ${ }^{g}$ German Center for Diabetes Research (DZD), Neuherberg, Germany \\ ${ }^{h}$ University of Hawaii Cancer Center, Honolulu, HI, USA \\ ${ }^{i}$ Department of Epidemiology, Erasmus Medical Centre, Rotterdam, the Netherlands \\ ${ }^{j}$ Division of Analytical Biosciences, Leiden Academic Centre for Drug Research, Leiden University, RA Leiden, the Netherlands \\ ${ }^{k}$ Department of Pathology \& Laboratory Medicine, University of Pennsylvania, Philadelphia, PA, USA \\ ${ }^{l}$ Center for Imaging of Neurodegenerative Diseases, Department of Radiology, San Francisco VA Medical Center/University of California San Francisco, San \\ Francisco, CA, USA \\ ${ }^{m}$ Duke Institute of Brain Sciences, Duke University, Durham, NC, USA \\ ${ }^{n}$ Department of Medicine, Duke University, Durham, NC, USA
}

Abstract

\begin{abstract}
Introduction: Bile acids (BAs) are the end products of cholesterol metabolism produced by human and gut microbiome co-metabolism. Recent evidence suggests gut microbiota influence pathological features of Alzheimer's disease (AD) including neuroinflammation and amyloid- $\beta$ deposition.

Method: Serum levels of 20 primary and secondary BA metabolites from the AD Neuroimaging Initiative $(n=1562)$ were measured using targeted metabolomic profiling. We assessed the association of BAs with the "A/T/N" (amyloid, tau, and neurodegeneration) biomarkers for AD: cerebrospinal fluid (CSF) biomarkers, atrophy (magnetic resonance imaging), and brain glucose metabolism $\left(\left[{ }^{18} \mathrm{~F}\right] \mathrm{FDG}\right.$ PET).
\end{abstract}

Results: Of 23 BAs and relevant calculated ratios after quality control procedures, three BA signatures were associated with CSF A $\beta_{1-42}$ ("A") and three with CSF p-tau181 ("T") (corrected $P<.05$ ).

The authors have declared that no conflict of interest exists.

${ }^{1}$ Equal contributors.

${ }^{2}$ Data used in preparation of this article were obtained from the Alzheimer's Disease Neuroimaging Initiative (ADNI) database (adni.loni. usc.edu). As such, the investigators within the ADNI contributed to the design and implementation of ADNI and/or provided data but did not participate in analysis or writing of this report. A complete listing of ADNI investigators can be found at http://adni.loni.usc.edu/wp-content/uploads/ how_to_apply/ADNI_Acknowledgement_List.pdf.

*Corresponding author. Tel.: +1 919684 2611; Fax: +1 9196817668.

**Corresponding author. Tel.: +1-317-963-7501; Fax: +1-317-9637547.

E-mail address: rima.kaddurahdaouk@duke.edu or Kaddu001@mc. duke.edu (R.K-D.) 
Furthermore, three, twelve, and fourteen BA signatures were associated with CSF t-tau, glucose metabolism, and atrophy ("N"), respectively (corrected $P<.05$ ).

Discussion: This is the first study to show serum-based BA metabolites are associated with "A/T/N" AD biomarkers, providing further support for a role of BA pathways in AD pathophysiology. Prospective clinical observations and validation in model systems are needed to assess causality and specific mechanisms underlying this association.

(C) 2018 Published by Elsevier Inc. on behalf of the Alzheimer's Association. This is an open access article under the CC BY-NC-ND license (http://creativecommons.org/licenses/by-nc-nd/4.0/).

Keywords: $\quad$ Metabolomics; Bile acid; Alzheimer's disease; Amyloid- $\beta$; CSF biomarkers; Brain glucose metabolism; PET; MRI; Gut-liver-brain axis

\section{Introduction}

Several metabolic perturbations are noted in Alzheimer's disease (AD), including failures associated with cholesterol metabolism [1-3], which has been associated with $\mathrm{AD}$ in multiple lines of research including physiological and epidemiological studies [3-5]. Bile acids (BAs) are synthesized from cholesterol and are involved in the digestion of lipids and absorption of fat-soluble vitamins [6]. Cholesterol is synthesized in the liver and its clearance involves BA production by gut microbiome and human co-metabolism. Changes in microbial gut populations can profoundly alter BA profiles and signaling [7-9]. BA synthesis can also be regulated by fat-soluble vitamins through repression of the rate-limiting enzyme cytochrome P450 7A (CYP7A1) [6]. Activation of the vitamin $D$ receptor is the mechanism by which vitamin $D$ affects BA homeostasis, whereas the action of vitamin A is mediated through the retinal $\mathrm{X}$ receptor/farnesoid $\mathrm{X}$ receptor heterodimer [6]. Vitamin-D-dependent regulation of BA synthesis may be an additional mechanism by which BAs influence $\mathrm{AD}$ pathophysiology as vitamin $\mathrm{D}$ is protective against risk of $\mathrm{AD}$ and cognitive impairments $[10,11]$.

BAs appear to play a role in the central nervous system $[12,13]$. Recent work suggests that microbial disturbances linked to BA profiles are implicated in neurodegenerative disorders [7-9,14-16]. The gut microbiota are involved in immune, neuroendocrine, and neural pathways $[8,9,17-$ 21], have been shown to regulate microglial maturation and function, and may contribute to $\mathrm{AD}$ [21,22].

Peripheral metabolic changes may influence central changes through the liver and gut-brain axis that includes commensal and pathogenic bacteria, through its interactions with the vagus nerve, changes in central nervous system functioning, the immune system [23,24], and hippocampal neurogenesis [25]. These signals are crucial for the regulation of energy, glucose homeostasis, and inflammation [26]. Gut microbiota are vital to the transformation of BAs through their deconjugation, dihydroxylation, and reconjugation [16]. The gut-brain biochemical axis of communication is just starting to be elucidated. Circulating BAs seem to provide an important mechanism for communication between the gut and the brain, and their alterations reflect gut dysbiosis $[8,9,18]$. Previous studies suggest that BAs are altered in mild cognitive impairment (MCI) and $\mathrm{AD}$ [27], and in the preceding article, we showed that increased levels of secondary cytotoxic BAs and their ratios to primary BAs were associated with $\mathrm{AD}$ and poor cognition (MahmoudianDehkordi et al., unpublished data, 2018). This supported the hypothesis that circulating BAs may contribute to $\mathrm{AD}$ pathogenesis. Research in $\mathrm{AD}$ animal models suggests a role for the gut microbiome in the development of amyloid- $\beta(\mathrm{A} \beta)$ pathology [28].

However, little work has been done in humans to link peripheral metabolic changes in cholesterol to central biomarkers related to $\mathrm{AD}$ including $\mathrm{A} \beta$ and tau accumulation, brain glucose metabolism, and structural atrophy. Therefore, we analyzed serum BA metabolites and their ratios from older adults with early-stage $\mathrm{AD}$ or who were at risk for $\mathrm{AD}$ from the Alzheimer's Disease Neuroimaging Initiative (ADNI) cohort.

We hypothesized that serum BA levels and their relevant ratios would associate with biomarkers of AD pathophysiology including neuroimaging (magnetic resonance imaging [MRI] and position emission tomography [PET]) and cerebrospinal fluid (CSF). The AD biomarkers were selected and defined consistent with the recent NIA-Alzheimer's Association Research Framework ("A/T/N") for AD biomarkers, which defines three general groups of biomarkers based on the nature of pathologic process that each measures [29-31]. This approach has several strengths including the focus on biological underpinnings of the disease rather than clinical phenotypes, which may have different pathophysiological substrates and making no assumptions based on the temporal order of the biomarkers included or their potential causal relationships. Biomarkers of $A \beta$ plaque ("A") are CSF $A \beta_{1-42}$ and cortical $\mathrm{A} \beta$ accumulation measured by florbetapir PET, biomarkers of fibrillary tau ("T") are CSF phosphorylated tau (p-tau), and biomarkers of neurodegeneration or neuronal injury ("N") are atrophy on MRI, glucose metabolism on FDG PET, and CSF total tau (t-tau).

\section{Methods}

\subsection{Study cohort}

Serum samples and data analyzed in the present report were obtained from ADNI. The initial phase (ADNI-1) 
was launched in 2003 to test whether serial MRI, PET, other biological markers, and clinical and neuropsychological assessment could be combined to measure the progression of MCI and early AD. ADNI-1 was extended to subsequent phases (ADNI-GO, ADNI-2, and ADNI-3) for follow-up for existing participants and additional new enrollments. Inclusion and exclusion criteria, clinical and neuroimaging protocols, and other information about ADNI can be found at www.adni-info.org [32,33]. Demographic information, raw neuroimaging scan data, apolipoprotein E (APOE) status, neuropsychological test scores, and clinical information are available and were downloaded from the ADNI data repository (www.loni.usc.edu/ADNI/). Written informed consent was obtained at the time of enrollment that included permission for analysis and data sharing, and consent forms were approved by each participating sites' Institutional Review Board.

\subsection{Quality control procedures of serum BA profiles}

Targeted metabolomics profiling was performed to identify and quantify concentrations of 20 BAs from serum samples using Biocrates Bile Acids Kit as described in detail in the companion article in this volume (MahmoudianDehkordi et al, 2018 [submitted]). In brief, morning serum samples from the baseline visit were collected and aliquoted as described in the ADNI standard operating procedures, with only fasting samples included in this study [32]. BA quantification was performed by liquid chromatography tandem mass spectrometry. Metabolites with $>40 \%$ of measurements below the lower limit of detection $(<$ LOD) were excluded. To assess the precision of the measured analytes, a set of blinded analytical replicates (24 pairs in ADNI-1 and 15 triples in ADNI-GO/2) were supplied by ADNI. Unblinded metabolite profiles went through further quality control (QC) checks. Validation of the kit was completed with acceptable interlaboratory and intralaboratory reproducibility as part of an international ring trial on the Bile Acids Kit from Biocrates AG, published in 2016 [34]. Interplate variability was monitored using a series of QC samples, including low, mid, and high QC that come with the kit, a study pool QC analyzed in triplicates on each plate, and the NIST SRM-1950 reference material in duplicate on each plate, and only analytes with $<30 \%$ technical reproducibility were included in subsequent analysis. The coefficient of variation (range 6.25, 21.49) and intraclass correlation coefficients (range 0.89, $0.99)$ are presented in Supplementary Table 1. The preprocessed data set included 15 BAs (5 BAs did not pass QC criteria) and 8 ratios. These selected ratios reflect enzymatic dysfunctions in the liver and changes in gut microbiome metabolism (see Fig. 1B and Section 2.3). The preprocessed BA values obtained from the QC step were adjusted for the effect of medication use (at baseline) on
BA levels (see Toledo et al. 2017 [35] for adjustment description details).

\subsection{Ratios reflective of conversion of BAs by gut microbiome}

We investigated eight selected ratios that are thought to be reflective of enzymatic activities in the liver and the gut microbiome to determine if these enzymatic processes in BA metabolism are associated with neuroimaging and CSF biomarkers of $\mathrm{AD}$. These ratios include

1. Cholic acid:chenodeoxycholic acid (CA:CDCA) ratio was selected to test if a possible shift in BA synthesis from the primary to the alternative BA pathways occurs in the liver.

2. Ratios of secondary to primary BAs (deoxycholic acid [DCA]:CA, glycolithocholic acid [GLCA]:CDCA, and taurolithocholic acid [TLCA]:CDCA) to examine differences in the gut microbiome enzymatic activity leading to altered production of secondary BAs. Because lithocholic acid (LCA) was excluded in QC steps, the GLCA:CDCA and TLCA:CDCA ratios were used as proxies for LCA:CDCA ratio.

3. GDCA:DCA and TDCA:DCA ratios were used to test if the observed secondary BA dysregulation is related to enzymatic differences related to conjugation.

\subsection{Neuroimaging processing}

\subsubsection{Magnetic resonance imaging}

T1-weighted brain MRI scans at baseline were acquired using a sagittal 3D MP-RAGE sequence following the ADNI MRI protocol [36,37]. As detailed in previous studies, FreeSurfer V5.1, a widely employed automated MRI analysis approach, was used to process MRI scans and extract whole brain and region-of-interest (ROI)-based neuroimaging endophenotypes including volumes and cortical thickness determined by automated segmentation and parcellation [38-40]. The cortical surface was reconstructed to measure thickness at each vertex. The cortical thickness was calculated by taking the Euclidean distance between the gray/white boundary and the gray/ CSF boundary at each vertex on the surface [41-43].

\subsubsection{Positron emission tomography}

Preprocessed $\left[{ }^{18} \mathrm{~F}\right]$ FDG and $\left[{ }^{18} \mathrm{~F}\right]$ florbetapir PET scans (co-registered, averaged, standardized image and voxel size, uniform resolution) were downloaded from the ADNI LONI site (http://adni.loni.usc.edu) as described in previously reported methods for acquisition and processing of PET scans from the ADNI sample $[38,44]$. For $\left[{ }^{18} \mathrm{~F}\right]$ FDG PET, scans were intensity-normalized using a pons ROI to create $\left[{ }^{18} \mathrm{~F}\right]$ FDG standardized uptake value ratio (SUVR) images. For 
A

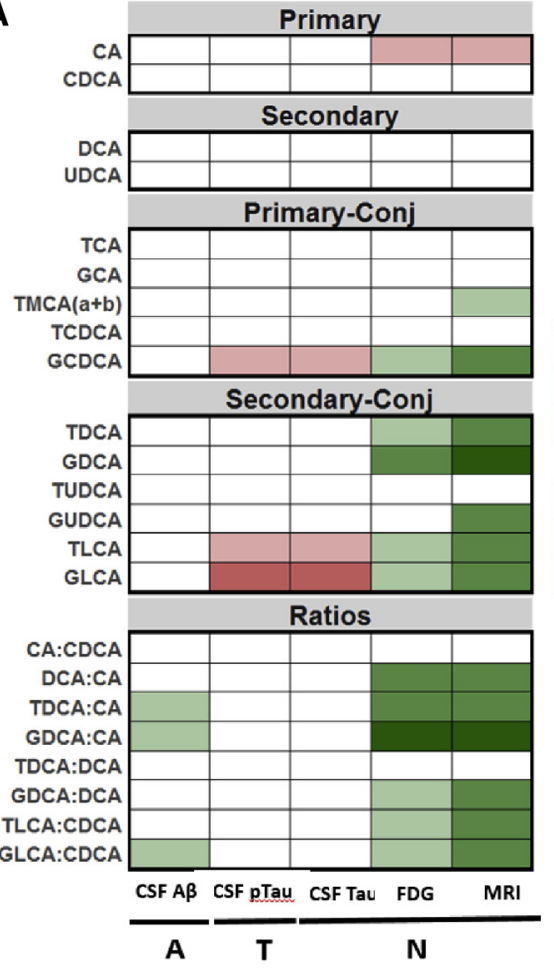

B

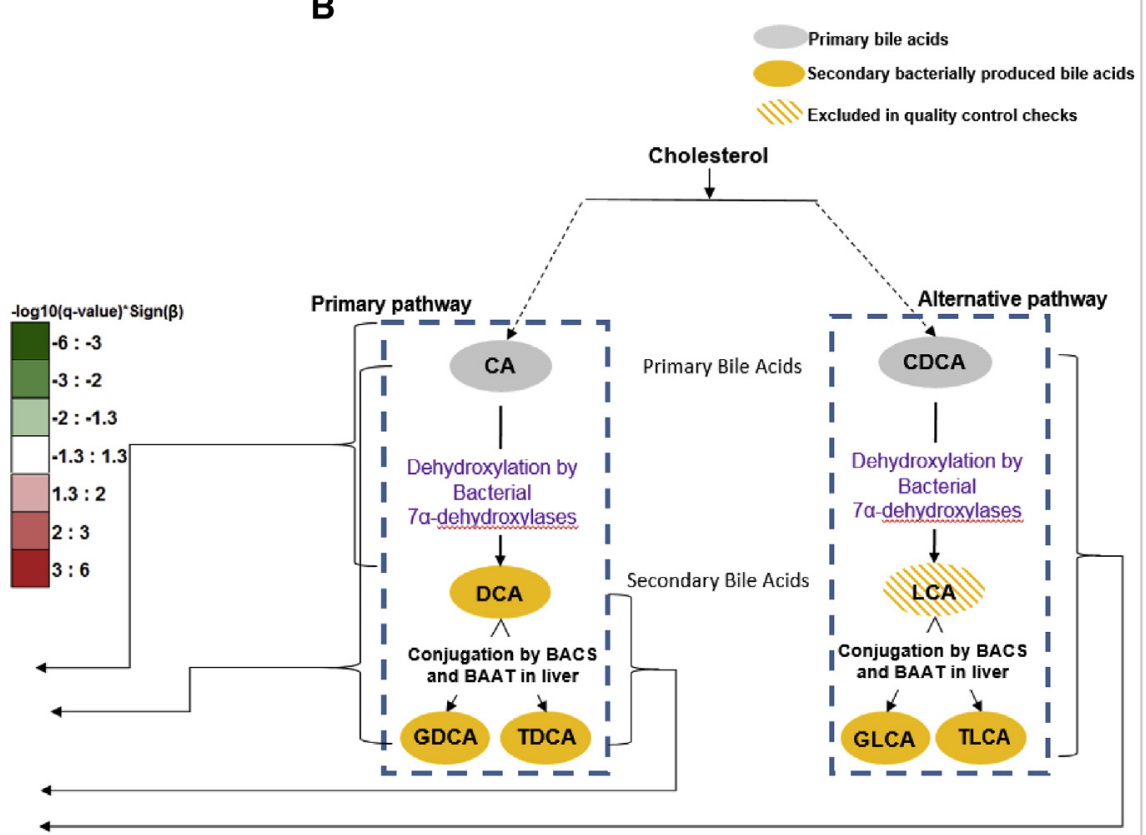

Fig. 1. Bile acids and their ratios reflective of gut microbiome and liver enzymatic activities and their correlation with ATN biomarkers for Alzheimer's disease. Heat map of q-values of association between bile acid profiles and the "A/T/N" biomarkers for $\mathrm{AD}(\mathrm{A}) . P$-values estimated from linear regression analyses were corrected for multiple testing using FDR ( $q$-value). Color code: white indicates q-value $>0.05$, reds indicate significant positive associations, and greens indicate significant negative associations. Several ratios were calculated to inform about possible enzymatic activity changes in AD (B). These ratios reflect (1) shift in bile acid metabolism from primary to alternative pathway, (2) changes in gut microbiome correlated with production of secondary bile acids, and (3) changes in glycine and taurine conjugation of secondary bile acids. LCA was excluded in prepossessing checks. Abbreviations: BAAT, amino acid N-acetyltransferase; BACS, bile acid-CoA synthetase; CA, cholic acid; CDCA, chenodeoxycholic acid; DCA, deoxycholic acid; GCA, glycocholic acid; GCDCA, glycochenodeoxycholic acid; GDCA, glycodeoxycholic acid; GLCA, glycolithocholic acid; GUDCA, glycoursodeoxycholic acid; LCA, lithocholic acid; TCA, taurocholic acid; TCDCA, taurochenodeoxycholic acid; TDCA, taurodeoxycholic acid; TLCA, taurolithocholic acid; TMCA, trimethoxycinnamic acid; TUDCA, tauroursodeoxycholic acid; UDCA, ursodeoxycholic acid; CSF, cerebrospinal fluid; MRI, magnetic resonance imaging; A $\beta$, amyloid- $\beta$.

$\left[{ }^{18} \mathrm{~F}\right]$ florbetapir PET, scans were intensity-normalized using a whole-cerebellum reference region to create SUVR images.

\section{5. $C S F A \beta_{1-42}$, t-tau, and $p$-tau ${ }_{181}$ biomarkers}

ADNI generated CSF biomarkers $\left(\mathrm{A} \beta_{1-42}, \mathrm{t}-\mathrm{tau}\right.$, and $\mathrm{p}$ $\operatorname{tau}_{181}$ ) in pristine aliquots of 2401 ADNI CSF samples using the validated and highly automated Roche Elecsys electrochemiluminescence immunoassays $[45,46]$ and the same reagent lot for each of these three biomarkers. The CSF biomarker data were downloaded from the ADNI LONI site (http://adni.loni.usc.edu).

\subsection{Statistical analyses}

\subsubsection{CSF biomarkers}

We performed a linear regression analysis using age, sex, study phase (ADNI-1 or ADNI-GO/2), body mass index (BMI), and $A P O E \& 4$ status as covariates, followed by false discovery rate (FDR)-based multiple comparison adjustment with the Benjamini-Hochberg procedure.

\subsubsection{ROI-based analysis of structural MRI and PET}

Mean hippocampal volume was used as an MRI-related phenotype. For FDG PET, a mean SUVR value was extracted from a global cortical ROI representing regions where patients with AD show decreased glucose metabolism relative to cognitively normal older participants from the full ADNI-1 cohort, normalized to pons [44]. For $\left[{ }^{18} \mathrm{~F}\right]$ florbetapir PET, a mean SUVR value was extracted using MarsBaR from a global cortical region generated from an independent comparison of ADNI-1 [11C] Pittsburgh compound B SUVR scans (regions where AD $>$ cognitively normal). We performed a linear regression analysis using age, sex, BMI, study phase (ADNI-1 or ADNI-GO/2), and APOE $\varepsilon 4$ status as covariates. For hippocampal volume, years of education, intracranial volume, and magnetic field strength were added as additional covariates. FDR-based multiple comparison adjustment with the Benjamini-Hochberg procedure was used because the $\mathrm{AD}$ biomarker phenotypes were strongly correlated with each other [47]. Not accounting for this high collinearity of dependent variables would lead to an overly stringent correction for multiple testing. 


\subsubsection{Whole-brain imaging analysis}

The SurfStat software package (www.math.mcgill.ca/ keith/surfstat/) was used to perform a multivariate analysis of cortical thickness to examine the effect of BA profiles on brain structural changes on a vertex-by-vertex basis using a general linear model (GLM) approach [43]. GLMs were developed using age, sex, years of education, intracranial volume, BMI, APOE \&4 status, and magnetic field strength as covariates. The processed FDG PET images were used to perform a voxel-wise statistical analysis of the effect of BA levels on brain glucose metabolism across the whole brain using SPM8 (www.fil.ion.ucl.ac.uk/spm/). We performed a multivariate regression analysis using age, sex, BMI, APOE \&4 status, and study phase (ADNI-1 or ADNI$\mathrm{GO} / 2$ ) as covariates. In the whole-brain surface-based analysis, the adjustment for multiple comparisons was performed using the random field theory correction method with $P<.05$ adjusted as the level for significance [48-50]. In the voxel-wise whole-brain analysis, the significant statistical parameters were selected to correspond to a threshold of $P<.05$ (FDR-corrected).

\section{Results}

\subsection{Study samples after $Q C$}

After QC procedures, 1562 ADNI participants with 23 BAs and their relevant ratio levels (15 BA metabolites and 8 ratios) at baseline (370 cognitively normal older adults, 98 significant memory concern, 284 early MCI, 505 late $\mathrm{MCI}$, and $305 \mathrm{AD}$ ) were available for analysis. Demographic information for the study population is presented in Table 1. Mean and standard errors of primary and secondary BAs stratified by clinical diagnosis are presented in Table 2.

\subsection{Biomarkers of amyloid- $\beta$ ( “A”)}

We used CSF $A \beta_{1-42}$ levels and a global cortical amyloid deposition of amyloid PET as biomarkers of A $\beta$. First, we evaluated whether BA profiles were associated with the CSF A $\beta_{1-42}$ biomarker by performing an association analysis for $15 \mathrm{BA}$ metabolites and 8 relevant ratios with $A P O E \varepsilon 4$ status as a covariate. As shown in Fig. 1A, after applying FDR-based multiple comparison correction, we identified three BA ratios significantly associated with CSF A $\beta_{1-42}$ levels. Regression coefficients of the three BA ratios of bacterially produced conjugated secondary BAs to primary BAs (GDCA:CA, TDCA:CA, and GLCA:CDCA) showed negative associations indicating higher levels were associated with lower CSF A $\beta_{1-42}$ values (CSF A $\beta_{1-42}$ positivity). However, global cortical amyloid deposition of amyloid PET was not significantly associated with any BA or their ratios after applying FDR-based multiple comparison correction. GDCA:CA was marginally associated with a global cortical amyloid load (uncorrected $P$-value $<.05$ ). Higher GDCA:CA levels were associated with greater amyloid deposition.

\subsection{Biomarkers of fibrillary tau (" $T$ ”)}

We used CSF phosphorylated tau (p-tau) levels as the biomarker of fibrillary tau. We investigated the association of $23 \mathrm{BAs}$ and their relevant ratios with CSF p-tau, with $A P O E \& 4$ status included as a covariate. We identified three significant associations (FDR-corrected $P<.05$ ) (Fig. 1A). For one conjugated primary BA metabolite (glycochenodeoxycholic acid [GCDCA]), higher GCDCA levels were associated with higher CSF p-tau values. For two bacterially produced conjugated secondary BA metabolites (GLCA and TLCA), higher levels were correlated with higher CSF p-tau values.

\subsection{Biomarkers of neurodegeneration or neuronal injury ( "N")}

We used atrophy on T1-weighted MRI, hypometabolism on FDG PET, and CSF total tau (t-tau) levels as biomarkers of neurodegeneration or neuronal injury.

\subsubsection{Structural MRI (atrophy)}

We investigated the association of BA metabolites and ratios with mean hippocampal volume with $A P O E \varepsilon 4$ status as

Table 1

Demographics of ADNI participants stratified by baseline diagnosis*

\begin{tabular}{|c|c|c|c|c|c|c|}
\hline Variable & $\mathrm{N}$ & $\mathrm{CN}(\mathrm{N}=370)$ & $\operatorname{SMC}(\mathrm{N}=98)$ & EMCI $(\mathrm{N}=284)$ & LMCI $(\mathrm{N}=505)$ & $\mathrm{AD}(\mathrm{N}=305)$ \\
\hline Sex: female, $n(\%)$ & 1562 & $190(51)$ & $56(57)$ & $130(46)$ & $139(39)$ & $139(46)$ \\
\hline BMI $\left(\mathrm{kg} / \mathrm{m}^{2}\right)$ & 1562 & $27.05(4.46)$ & $28.22(6.24)$ & $28.06(5.41)$ & $26.54(4.25)$ & $25.83(4.69)$ \\
\hline$A P O E \varepsilon 4$ status, $(+) n(\%)$ & 1562 & $104(28)$ & $32(33)$ & $121(43)$ & $273(54)$ & $202(66)$ \\
\hline $\mathrm{CSF} A \beta_{1-42}$ & 1112 & $1342.2(663.7)$ & $1385.7(605.0)$ & $1175.1(569.3)$ & $896.5(502.7)$ & $683.0(394.6)$ \\
\hline
\end{tabular}

Abbreviations: ADNI, Alzheimer's Disease Neuroimaging Initiative; APOE, apolipoprotein E; AD, Alzheimer's disease; BMI, body mass index; CN, cognitively normal; EMCI, early mild cognitive impairment; LMCI, late mild cognitive impairment; SMC, subjective memory complaint; CSF: cerebrospinal fluid; $\mathrm{A} \beta$, amyloid- $\beta$.

*Data are reported as mean $(\mathrm{SD})$ unless otherwise indicated. 
Table 2

Levels of primary and secondary bile acids measured in the ADNI cohort stratified by clinical diagnosis*

\begin{tabular}{llllllll}
\hline Bile acid & Category & $\mathrm{N}^{\dagger}$ & $\mathrm{CN}(\mathrm{N}=370)$ & $\mathrm{SMC}(\mathrm{N}=98)$ & EMCI $(\mathrm{N}=284)$ & $\mathrm{LMCI}(\mathrm{N}=505)$ & $\mathrm{AD}(\mathrm{N}=305)$ \\
\hline CA & Primary & 1446 & $0.221(0.024)$ & $0.245(0.093)$ & $0.155(0.021)$ & $0.192(0.021)$ & $0.135(0.025)$ \\
CDCA & Primary & 1357 & $0.285(0.042)$ & $0.315(0.128)$ & $0.241(0.034)$ & $0.288(0.033)$ & $0.216(0.033)$ \\
GCA & Primary conjugated & 1463 & $0.236(0.019)$ & $0.269(0.046)$ & $0.234(0.021)$ & $0.239(0.014)$ & $0.297(0.037)$ \\
GCDCA & Primary conjugated & 1464 & $0.658(0.035)$ & $0.702(0.078)$ & $0.724(0.059)$ & $0.710(0.037)$ & $0.806(0.049)$ \\
TCA & Primary conjugated & 1020 & $0.068(0.008)$ & $0.090(0.029)$ & $0.057(0.006)$ & $0.068(0.006)$ & $0.066(0.009)$ \\
TCDCA & Primary conjugated & 1426 & $0.090(0.006)$ & $0.114(0.025)$ & $0.088(0.007)$ & $0.091(0.006)$ & $0.097(0.008)$ \\
TMCA & Primary conjugated & 1146 & $0.012(0.001)$ & $0.007(0.001)$ & $0.011(0.001)$ & $0.014(0.002)$ & $0.014(0.002)$ \\
DCA & Secondary & 1445 & $0.526(0.041)$ & $0.520(0.068)$ & $0.574(0.043)$ & $0.529(0.026)$ & $0.627(0.045)$ \\
UDCA & Secondary & 1111 & $0.065(0.007)$ & $0.044(0.008)$ & $0.072(0.011)$ & $0.091(0.010)$ & $0.087(0.012)$ \\
GDCA & Secondary conjugated & 1439 & $0.440(0.034)$ & $0.462(0.063)$ & $0.488(0.038)$ & $0.502(0.031)$ & $0.672(0.054)$ \\
TDCA & Secondary conjugated & 1430 & $0.058(0.006)$ & $0.077(0.019)$ & $0.059(0.005)$ & $0.065(0.005)$ & $0.077(0.006)$ \\
GLCA & Secondary conjugated & 1037 & $0.027(0.002)$ & $0.025(0.003)$ & $0.034(0.003)$ & $0.030(0.002)$ & $0.039(0.003)$ \\
TLCA & Secondary conjugated & 1008 & $0.005(0.0002)$ & $0.004(0.001)$ & $0.005(0.0003)$ & $0.005(0.0003)$ & $0.006(0.0005)$ \\
GUDCA & Secondary conjugated & 1401 & $0.115(0.010)$ & $0.093(0.015)$ & $0.114(0.012)$ & $0.129(0.012)$ & $0.136(0.015)$ \\
TUDCA & Secondary conjugated & 1369 & $0.008(0.001)$ & $0.008(0.001)$ & $0.008(0.001)$ & $0.008(0.001)$ & $0.008(0.001)$ \\
\hline
\end{tabular}

Abbreviations: AD, Alzheimer's disease; CN, cognitively normal; EMCI, early mild cognitive impairment; LMCI, late mild cognitive impairment; SMC, subjective memory complaint; CA, cholic acid; CDCA, chenodeoxycholic acid; DCA, deoxycholic acid; GCA, glycocholic acid; GCDCA, glycochenodeoxycholic acid; GDCA, glycodeoxycholic acid; GLCA, glycolithocholic acid; GUDCA, glycoursodeoxycholic acid; LCA, lithocholic acid; TCA, taurocholic acid; TCDCA, taurochenodeoxycholic acid; TDCA, taurodeoxycholic acid; TLCA, taurolithocholic acid; TMCA, trimethoxycinnamic acid; TUDCA, tauroursodeoxycholic acid; UDCA, ursodeoxycholic acid.

*Values represent $\mu \mathrm{M}$ in mean (standard error of the mean).

${ }^{\dagger}$ Number of nonmissing measurements.

a covariate. Among 23 BA characteristics, 14 BAs/ratios were significantly associated with hippocampal volume after controlling for multiple testing using FDR (Fig 1A; corrected $P<.05$ ). For one primary BA metabolite, lower CA levels were associated with decreased hippocampal volume. However, for two conjugated primary BA metabolites [GCDCA and $\operatorname{TMCA}(\mathrm{a}+\mathrm{b})]$ and five bacterially produced conjugated secondary BA metabolites (GDCA, GLCA, GUDCA, TDCA, and TLCA), higher BA levels were associated with decreased hippocampal volume. In addition, higher levels of six ratios of bacterially produced secondary BA metabolite to primary BA metabolite (DCA:CA, GDCA:CA, TDCA:CA, GDCA:DCA, GLCA:CDCA, and TLCA:CDCA) were associated with decreased hippocampal volume.

Among the 14 significant BA signatures, six BA profiles were significantly associated with CSF $A \beta_{1-42}$ biomarker ("A") or CSF p-tau biomarker ("T"). For the six BA profiles, we performed a detailed whole-brain surface-based analysis using multivariate regression models and assessed their effects on whole-brain cortical thickness in an unbiased way. We identified significant associations for all six BA profiles (cluster-wise threshold of random field theory-corrected $P<.05$ ), which showed consistent patterns in the associations of CSF $A \beta_{1-42}$ or p-tau levels (Fig. 2). Higher levels of a conjugated primary BA (GCDCA) were significantly associated with reduced cortical thickness especially in the bilateral entorhinal cortices. Increased levels of one bacterially produced conjugated secondary BA metabolite (GLCA) and two ratios of bacterially produced secondary BA metabolites to primary BA metabolites (GDCA:CA and GLCA:CDCA) were significantly associated with reduced cortical thickness in the bilateral frontal, parietal, and temporal lobes including the entorhinal cortex. For one bacterially produced conjugated secondary BA metabolite (TLCA) and one ratio of a bacterially produced secondary BA metabolite to a primary BA metabolite (TDCA:CA), increased levels were associated with reduced cortical thickness in a widespread pattern, especially in the bilateral frontal, parietal, and temporal lobes.

\subsubsection{FDG PET (brain glucose metabolism)}

We performed an association analysis for $23 \mathrm{BA}$ and ratios with global cortical glucose metabolism measured by FDG PET scans across 1066 participants with both FDG PET scans and BA measurements. The association testing including $A P O E \& 4$ status as a covariate identified $12 \mathrm{BA}$ characteristics as significantly associated with brain glucose metabolism after controlling for multiple testing using FDR (Fig. 1A; corrected $P<.05$ ). For one primary BA metabolite, lower CA levels were associated with reduced glucose metabolism. In contrast, for one conjugated primary BA metabolite (GCDCA), four bacterially produced conjugated secondary BA metabolites (GDCA, GLCA, TDCA, and TLCA), and six ratios of bacterially produced secondary BA metabolites to primary BA metabolites (DCA:CA, GDCA:CA, TDCA:CA, GDCA:DCA, GLCA:CDCA, and TLCA:CDCA), higher BA ratio levels were associated with reduced glucose metabolism.

In addition, in an unbiased way, we performed a detailed whole-brain analysis to determine the effect of BAs on brain glucose metabolism on a voxel-wise level for six BAs and ratios (GCDCA, GLCA, TLCA, GDCA:CA, TDCA:CA, and GLCA:CDCA) that were significantly associated with 

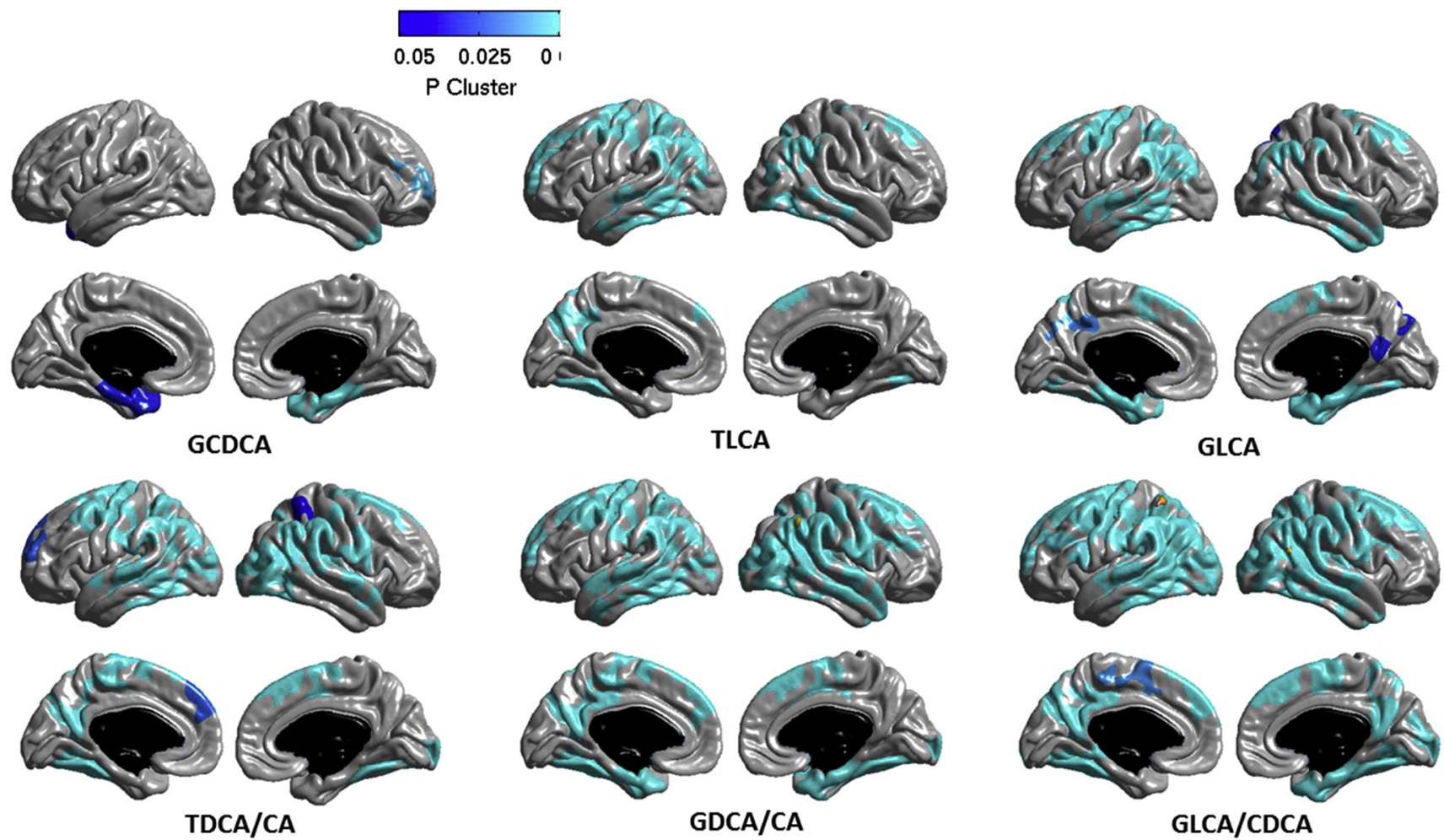

TLCA

GLCA
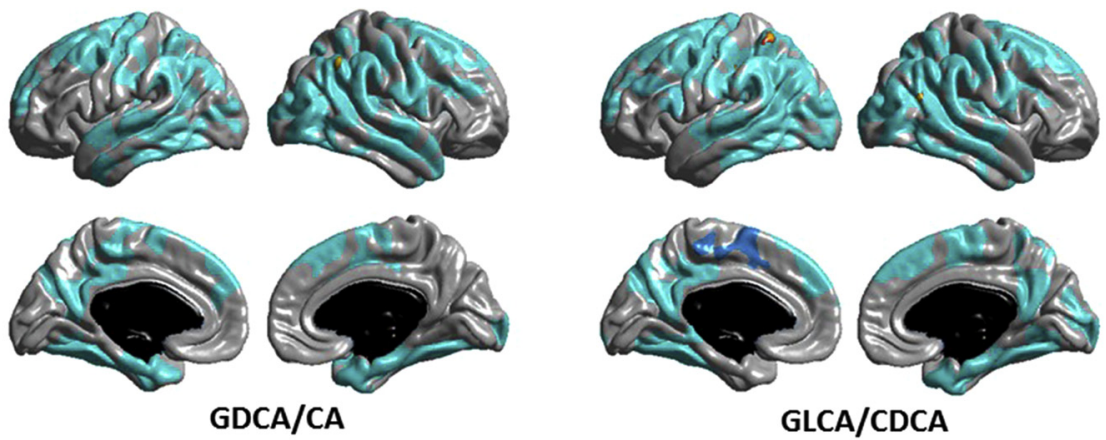

Fig. 2. Whole-brain surface-based imaging analysis. A whole-brain multivariate analysis of cortical thickness across the brain surface was performed to visualize the topography of the association of bile acid profiles with the brain structure in an unbiased manner. For a surface-based analysis of levels of CDCA, TLCA, GLCA, TDCA:CA, GDCA:CA, and GLCA:CDCA, statistical maps were thresholded using a random field theory for a multiple testing adjustment to a corrected significance level of 0.05 . The $P$-value for clusters indicates significant corrected $P$ values with the lightest blue color. Higher GCDCA levels were significantly associated with reduced cortical thickness especially in bilateral entorhinal cortices. Increased GLCA, GDCA:CA, and GLCA:CDCA levels were significantly associated with reduced cortical thickness in the bilateral frontal, parietal, and temporal lobes including the entorhinal cortex. For TLCA and TDCA:CA, increased levels were associated with reduced cortical thickness in a widespread pattern, especially in the bilateral frontal, parietal, and temporal lobes. Abbreviations: CA, cholic acid; CDCA, chenodeoxycholic acid; GCDCA, glycochenodeoxycholic acid; GDCA, glycodeoxycholic acid; GLCA, glycolithocholic acid; TDCA, taurodeoxycholic acid; TLCA, taurolithocholic acid.

both CSF A $\beta_{1-42}$ and p-tau biomarkers, FDG metabolism, and hippocampal volume. We identified significant associations for all six BA profiles (cluster-wise threshold of FDRcorrected $P<.05$ ), which showed consistent patterns in the associations of CSF A $\beta_{1-42}$ or p-tau levels and structural atrophy (Fig. 3). Higher levels of a conjugated primary bile acid GCDCA were significantly associated with reduced glucose metabolism especially in the bilateral hippocampi, which showed consistent patterns with the associations of cortical thickness. Increased levels of one bacterially produced conjugated secondary BA metabolite (GLCA) and one ratio of a bacterially produced secondary BA metabolite to a primary BA metabolite (GLCA:CDCA) were significantly associated with reduced glucose metabolism in the bilateral temporal and parietal lobes. Lower TLCA levels, a bacterially produced conjugated secondary BA metabolite, were associated with increased glucose metabolism in the left temporal lobe. For two ratios (GDCA:CA and TDCA:CA) of bacterially produced secondary BA metabolite to a primary BA metabolite, higher ratio levels were significantly associated with reduced glucose metabolism in a widespread pattern, especially in the bilateral frontal, parietal, and temporal lobes.

\subsubsection{CSF total tau (t-tau)}

We evaluated whether $23 \mathrm{BAs}$ and ratios were associated with the CSF t-tau including APOE $\varepsilon 4$ status as a covariate. We identified three significant associations after controlling for multiple testing using FDR (corrected $P<.05$ ) (Fig. 1). Higher levels of GCDCA, a conjugated primary BA metabolite, and GLCA and TLCA, bacterially produced secondary BA metabolites, were associated with higher CSF t-tau values.

\section{Discussion}

In this report, we analyzed serum-based BA profiles in the ADNI cohort to investigate the relationship between peripheral metabolic measures and central biomarkers for $\mathrm{AD}$ pathophysiology based on the recently proposed framework ("A/T/N") [30]. Our results showed that altered BA profiles were significantly associated with structural and functional changes in the brain as noted by larger atrophy and reduced glucose metabolism ("N"). Furthermore, altered BA profiles were significantly associated with three CSF biomarkers including $A \beta 1-42$, t-tau, and p-tau. Three ratios of primary BAs to secondary BAs were associated with lower CSF 

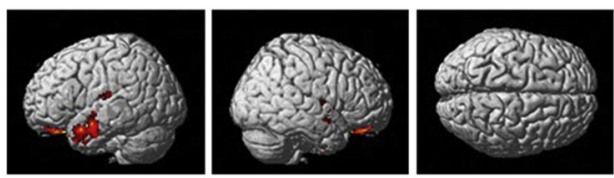

GCDCA
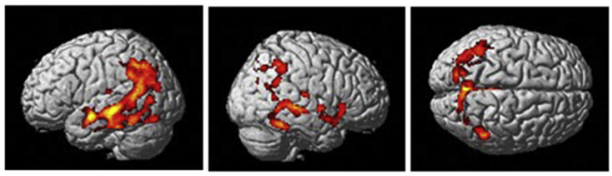

GLCA
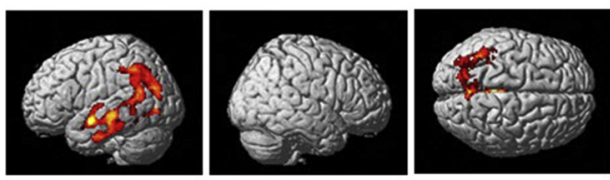

TLCA
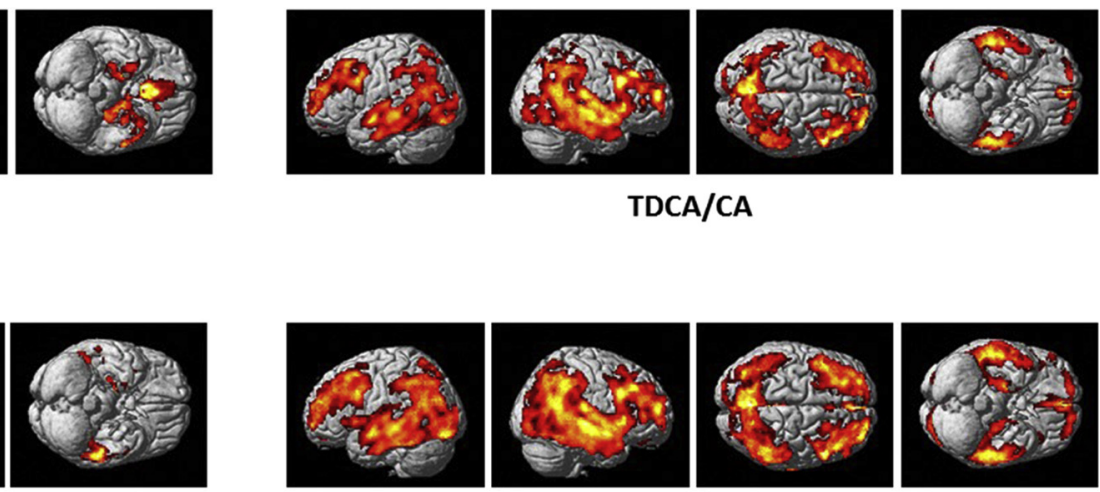

GDCA/CA
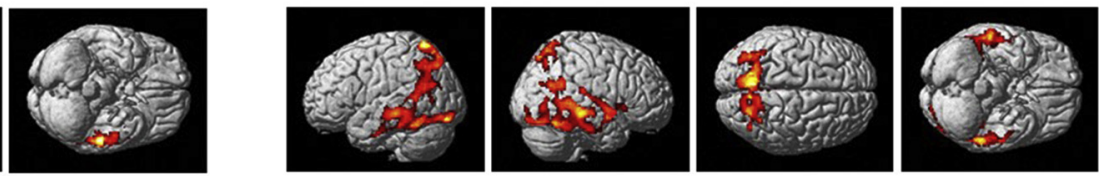

GLCA/CDCA

Fig. 3. Whole-brain voxel-based imaging analysis. A whole-brain multivariate analysis of glucose metabolism was performed to visualize the topography of the association of bile acid profiles with glucose metabolism in an unbiased manner. For a voxel-based analysis of FDG-PET scans, we identified significant associations (cluster-wise threshold of FDR-corrected $P<.05$ ). Higher GCDCA levels were significantly associated with reduced glucose metabolism especially in the bilateral hippocampi. Increased GLCA and GLCA:CDCA levels were significantly associated with reduced glucose metabolism in the bilateral temporal and parietal lobes. Lower TLCA levels were associated with increased glucose metabolism in the left temporal lobe. For two ratios (GDCA:CA and TDCA:CA), higher ratio levels were significantly associated with reduced glucose metabolism in a widespread pattern, especially in the bilateral frontal, parietal, and temporal lobes. Abbreviations: CA, cholic acid; CDCA, chenodeoxycholic acid; GCDCA, glycochenodeoxycholic acid; GDCA, glycodeoxycholic acid; GLCA, glycolithocholic acid; TDCA, taurodeoxycholic acid; TLCA, taurolithocholic acid; PET, positron emission tomography.

$\mathrm{A} \beta_{1-42}$ levels (amyloid- $\beta$ positivity) ("A") as well as reduced cortical glucose metabolism and larger structural atrophy (GDCA:CA, TDCA:CA, and GLCA:CDCA). One conjugated primary BA profile (GCDCA) and two bacterially produced conjugated secondary BAs (GLCA and TLCA) were associated with higher CSF p-tau values ("T") as well as higher CSF t-tau values, reduced glucose metabolism, and larger structural atrophy.

Whether the gut microbiome directly influences AD pathogenesis remains unknown; however, it does appear to influence $A \beta$, fibrillary tau, and neurodegeneration. To our knowledge, this is the first study to systematically link markers of the gut microbiome and liver function to ADrelated structural and functional neuroimaging biomarkers as well as biomarkers of $A \beta$ and tau burden.

Three core CSF biomarkers (A $\beta_{1-42}, \mathrm{t}$-tau, and $\mathrm{p}$-tau) reflect $\mathrm{AD}$ pathology and can be used to reliably diagnose $\mathrm{AD}$ and identify MCI, a prodromal stage of $\mathrm{AD}$, with high diagnostic accuracy [51,52]. Previous studies showed that patients with $\mathrm{AD}$ have a substantial reduction in CSF A $\beta_{1-42}$ and a marked increase in levels of CSF t-tau and p-tau [5356]. We observed that higher levels of TDCA:CA, GDCA:CA, and GLCA:CDCA were associated with decreased levels of CSF $A \beta_{1-42}$, and higher levels of GCDCA, TLCA, and GLCA were associated with increased levels of CSF t-tau and p-tau.
MRI is widely used to investigate structural changes in MCI and AD [57-59]. We observed lower levels of CA and higher levels of GCDCA, TMCA $(a+b)$, GDCA, GLCA, GUDCA, TDCA, TLCA, DCA:CA, GDCA:CA, TDCA:CA, GDCA:DCA, GLCA:CDCA, and GCDCA:CDCA were associated with greater brain atrophy. Significant regional effects were observed particularly in the bilateral inferior parietal gyri cortices, hippocampi, and temporal lobes including the entorhinal cortex. The hippocampus and entorhinal cortex are affected early in $\mathrm{AD}$, and the decrease in hippocampal volume accelerates as AD progresses. Significant thinning of the cortical surface reflects atrophy in the temporal, parietal, and frontal lobes and has been shown in MCI and AD [57-59]. Reduced cortical thickness in the temporal cortex as a measure of brain atrophy rate has shown promise in predicting MCI to AD progression [57].

Lower CA and higher TDCA, GDCA, GCDCA, GLCA, TLCA, DCA:CA, GDCA:CA, TDCA:CA, GDCA:DCA, GLCA:CDCA, and TLCA:CDCA levels were associated with reduced global glucose metabolism in the brain. The significant regional effect on brain glucose metabolism was observed particularly in the bilateral hippocampi for GCDCA, in the temporal and parietal lobes for GLCA, TLCA, and GLCA/CDCA, and in a widespread pattern including the bilateral temporal, parietal, and frontal lobes 
for GDCA:CA and TDCA:CA. Patients with AD have shown significant glucose metabolism reduction in the temporal lobes, parietal lobes, and then the frontal lobes with increasing severity of AD [60-62].

The observed pattern of the association between changes in brain structure and glucose metabolism as well as CSF biomarkers with specific BAs and ratios indicates a potential mechanistic connection between peripheral and central biochemical changes. Our results strongly suggest gutliver-brain axis involvement in $\mathrm{AD}$, neurodegeneration, and brain dysfunction. Both liver function and gut microbiome activity are impacted in $\mathrm{AD}$, and these changes seem to occur at the earliest stages of disease. Despite this strong pattern of associations, the specific mechanism and causal directionality remains to be determined.

We hypothesized that altered gut microbiota play an important role. This is supported by several lines of research connecting the gut microbiota and AD pathology. Alterations in the gut microbiota and an increase in gut permeability may lead to dysfunction in the hippocampus $[63,64]$ and the development of insulin resistance, which correlates with AD pathogenesis [65-67]. It has been hypothesized that increased gut permeability allows bacteria-derived amyloids from the gastrointestinal tract to accumulate at the systemic and brain level [68]. This in turn could lead to the upregulation of proinflammatory microRNA-34a, and as a consequence, downregulation of TREM2 leading to the accumulation of $A \beta_{42}$. $[67,68]$.

Results from animal studies demonstrate that increased input of BAs significantly inhibits two of the major phyla in the human gut microbiome, Bacteroidetes and Actinobacteria [69]. Bacterial taxonomic composition of fecal samples revealed differences in bacterial abundance including decreased Firmicutes, increased Bacteroidetes, and decreased Bifidobacterium (phylum Actinobacteria) in the microbiome of patients with $\mathrm{AD}$ relative to ageand sex-matched controls. Furthermore, these differences in bacterial abundance correlated with CSF biomarkers including $A \beta_{42} / A \beta_{40}$ and $p$-tau/A $\beta_{42}$. Even in the ageand sex-matched controls (no dementia diagnosis), there was a similar relationship between the same bacteria that were either more or less abundant in $\mathrm{AD}$ and markers of tau and amyloid [70]. In another study, an increased abundance of proinflammatory bacteria (Escherichia/Shigella) and a decreased abundance of anti-inflammatory bacteria (Eubacterium rectale) were noted in cognitively impaired older adults with evidence of amyloid deposition on PET imaging compared to those who were amyloid negative [71]. These results lend further support to the link between gut microbiota and brain amyloidosis. Gut microbiota have been associated with the accumulation of amyloid plaques in a mouse model of $\mathrm{AD}$. A transgenic $\mathrm{AD}$ mouse model generated under germ-free conditions had dramatic reductions in cerebral $\mathrm{A} \beta$ pathology compared with control animals with normal intestinal microbiota, whereas colonization of germ-free AD mice with microbiota har- vested from conventionally raised $\mathrm{AD}$ mice significantly increased $A \beta$ pathology [28].

The association between BA cytotoxicity and the generation of reactive oxygen species (ROS) is well documented [72-76]. Others have proposed that mitochondrial ROS production plays an important role in brain metabolic signaling [77,78]. Some of the mechanisms by which mitochondrial dysfunction leads to neuronal degeneration in $\mathrm{AD}$ include ROS generation and activation of mitochondrial permeability transition $[79,80]$, suggesting a crucial role for oxidative stress in the pathophysiology of AD. Hyperphosphorylation of tau proteins has been linked to oxidation through the microtubule-associated protein kinase pathway [81]. In our analyses, three cytotoxic BAs (GCDCA, GLCA, and TLCA) correlated with higher biomarker levels of fibrillary tau and neurodegeneration/ neuronal injury.

Hydrophobic BAs, such as CDCA, are known to damage biological membranes [82], whereas hydrophilic BAs, such as UDCA and TUDC, are inhibitors of apoptosis via their ability to stabilize mitochondrial membranes [83,84]. Impairment of mitochondrial function is likely one of the vital ways in which BAs cause cellular dysfunctions [8588]. Decreased mitochondrial membrane potential has been associated with increasing concentrations of the following BAs: LCA, DCA, UDCA, CDCA, GCDC, and taurochenodeoxycholic (TCDC) acid [85].

\subsection{Limitations}

The ADNI study is observational by design making it difficult to control for confounding as well as to determine directionality of associations and causal pathways. For example, the population of the gut is affected by a plethora of factors including geography, lifetime immunological experience, and environmental factors, which could play important yet currently unknown roles in the pathogenesis of $\mathrm{AD}$. Experimental studies are needed to understand the mechanistic role of $\mathrm{BA}$ in the development of $\mathrm{AD}$-related pathology as well as to disentangle cause and effect. Medication use was extensively explored as a potential confounder (Supplementary Fig. 2). Overall, our key findings remained significant after adjustment for medication use, although effects were attenuated. In addition, further studies are warranted to validate our findings in independent cohorts. In this article, we focus on secondary BAs that are only known to be synthesized by bacterial enzymes. It is possible that there might be an alternative route to their synthesis that is yet to be discovered. These additional regulatory mechanisms could be responsible for the observed changes.

Currently, no large-scale clinical studies (such as ADNI) have collected fecal materials to interrogate changes in the gut microbiome composition. Future studies we are planning in coordination with large $\mathrm{AD}$ centers will enable collection of matching blood and fecal materials where the 
composition of the gut microbiome across the trajectory of disease can be investigated. There is an urgent need to elucidate mechanistic links between gut microbial metabolism and brain function in AD. Existing studies elucidating relations between gut microbial metabolism and the human metabolome have mainly used statistical analyses to correlate individual compounds with microbial taxa [89,90]. However, correlation does not necessarily equal causation as the microbiome may change due to AD-related metabolic pathology including weight loss. Longitudinal studies that capture both the metagenome and metabolome along with fecal samples and dietary information are needed to begin to clarify the relationship between BA metabolism, gut dysbiosis, and AD.

\section{Conclusions}

This is the first study to our knowledge to demonstrate an association between altered BA profiles and $\mathrm{A} \beta$, tau, and neurodegeneration biomarkers of $\mathrm{AD}$ pathophysiology. Although our results provide further evidence implicating $\mathrm{BA}$ signaling in $\mathrm{AD}$, the causal pathway remains to be systematically investigated by prospective clinical studies and experimental manipulations in model systems. Future metagenomics studies are also needed to define the relationship between BAs, host factors including genetics, and bacterial community composition within an individual across time. Building on present results, these investigations are needed to achieve a mechanistic understanding of the role of gut bacteria and $\mathrm{BAs}$ in relation to $\mathrm{AD}$ pathophysiology. If a causal role can be demonstrated in future research, BA signaling pathways may lead to the identification of metabolites that are protective against $\mathrm{AD}$ and could foster novel therapeutic strategies.

\section{Acknowledgments}

Authors' contributions: K.N., S.MD, and M.A. had full access to all the data in the study and take responsibility for the integrity of the data and the accuracy of the data analysis. M.A. and G.K. contributed to statistical analyses. Data management and medication term mapping were done by C.B. R.K.-D. contributed to concept and design and led the concept and design team that included all co-authors. Drafting of the manuscript was done by K.N., A.K.-P., A.J.S., and R.K.-D. Biochemical, genomics, and medications integration were done by G.K., R.B., X.H., and S.L.R. Alzheimer's Disease Neuroimaging Initiative (see note) contributed to data deposition. Alzheimer's Disease Metabolomics Consortium (see note) contributed to harmonization of methods. G.L. contributed to technical, bibliographic research, and/or material support. Biochemical interpretation was done by R.B., X.H., and R.K.-D. Critical revision of the manuscript for important intellectual content was done by A.J.S., P.M.D., and R.K.-D.; R.K.-D obtained funding. Supervision was done by J.Q.T., L.M.S., M.W.W., P.M.D., A.J.S., G.K., and R.K.-D.

The Alzheimer's Disease Neuroimaging Initiative (ADNI): Data used in the preparation of this article were obtained from the ADNI database (http://adni.loni.usc.edu). As such, the investigators within the ADNI contributed to the design and implementation of ADNI and/or provided data but did not participate in analysis or writing of this report. A complete listing of ADNI investigators can be found at http://adni.loni.usc.edu/wp-content/uploads/how_to_apply/ ADNI_Acknowledgement_List.pdf.

Funding/Support: Funding for ADMC (Alzheimer's Disease Metabolomics Consortium, led by R.K.-D. at Duke University) was provided by the National Institute on Aging grant R01AG046171, a component of the Accelerated Medicines Partnership for AD (AMP-AD) Target Discovery and Preclinical Validation Project (https://www.nia.nih.gov/res earch/dn/amp-ad-target-discovery-and-preclinical-validation -project) and the National Institute on Aging grant RF1 AG0151550, a component of the $\mathrm{M}^{2} \mathrm{OVE}-\mathrm{AD}$ Consortium (Molecular Mechanisms of the Vascular Etiology of ADConsortium https://www.nia.nih.gov/news/decoding-molecu lar-ties-between-vascular-disease-and-alzheimers). Data collection and sharing for this project was funded by the Alzheimer's Disease Neuroimaging Initiative (ADNI) (National Institutes of Health Grant U01 AG024904) and DOD ADNI (Department of Defense award number W81XWH-12-20012). ADNI is funded by the National Institute on Aging, the National Institute of Biomedical Imaging and Bioengineering, and through generous contributions from the following: AbbVie; Alzheimer's Association; Alzheimer's Drug Discovery Foundation; Araclon Biotech; BioClinica, Inc.; Biogen; Bristol-Myers Squibb Company; CereSpir, Inc.; Eisai Inc.; Elan Pharmaceuticals, Inc.; Eli Lilly and Company; EuroImmun; F. Hoffmann La Roche Ltd and its affiliated company Genentech, Inc.; Fujirebio; GE Healthcare; IXICO Ltd.; Janssen Alzheimer Immunotherapy Research \& Development, LLC.; Johnson \& Johnson Pharmaceutical Research \& Development LLC.; Lumosity; Lundbeck; Merck \& Co., Inc.; Meso Scale Diagnostics, LLC.; NeuroRx Research; Neurotrack Technologies; Novartis Pharmaceuticals Corporation; Pfizer Inc.; Piramal Imaging; Servier; Takeda Pharmaceutical Company; and Transition Therapeutics. The Canadian Institutes of Health Research is providing funds to support ADNI clinical sites in Canada. Private sector contributions are facilitated by the Foundation for the National Institutes of Health (www. fnih.org). The grantee organization is the Northern California Institute for Research and Education, and the study is coordinated by the Alzheimer's Disease Cooperative Study at the University of California, San Diego. ADNI data are disseminated by the Laboratory for Neuro Imaging at the University of Southern California.

The work of various consortium investigators is also supported by various NIA grants [U01 AG024904-09S4, P50NS053488, R01AG19771, P30AG10133, P30AG10124, R03AG054936, 
and K01AG049050], the National Library of Medicine [R01LM011360, R01LM012535, and R00LM011384], and the National Institute of Biomedical Imaging and Bioengineering [R01EB022574]. Additional support came from Helmholtz Zentrum, the Alzheimer's Association, the Indiana Clinical and Translational Science Institute, and the Indiana University-IU Health Strategic Neuroscience Research Initiative.

Role of the funder/sponsor: Funders (listed previously) had no role in the design and conduct of the study; collection, management, analysis, and interpretation of the data; preparation, review, or approval of the manuscript; and decision to submit the manuscript for publication.

Additional contributions: The authors are grateful to Lisa Howerton for administrative support and the numerous ADNI study volunteers and their families.

Disclosures: J.Q.T. may accrue revenue in the future on patents submitted by the University of Pennsylvania, wherein he is a co-inventor and he received revenue from the sale of Avid to Eli Lily as a co-inventor on imaging-related patents submitted by the University of Pennsylvania. L.M.S. receives research funding from NIH (U01 AG024904, R01 MH 098260, R01 AG 046171, and 1RF AG 051550) and MJ Fox Foundation for PD Research and is a consultant for Eli Lilly, Novartis, and Roche; and he provides QC over-sight for the Roche Elecsys immunoassay as part of responsibilities for the ADNI study. A.J.S. reports investigator-initiated research support from Eli Lilly unrelated to the work reported here. He has received consulting fees and travel expenses from Eli Lilly and Siemens Healthcare and is a consultant to Arkley BioTek. He also receives support from Springer publishing as an editor in chief of Brain Imaging and Behavior. M.W.W. reports stock/ stock options from Elan, Synarc, travel expenses from Novartis, Tohoku University, Fundacio Ace, Travel eDreams, MCI Group, NSAS, Danone Trading, ANT Congress, NeuroVigil, CHRU-Hopital Roger Salengro, Siemens, AstraZeneca, Geneva University Hospitals, Lilly, University of California, San Diego-ADNI, Paris University, Institut Catala de Neurociencies Aplicades, University of New Mexico School of Medicine, Ipsen, Clinical Trials on Alzheimer's Disease, Pfizer, and AD PD meeting. P.M.D. has received research grants and advisory/speaking fees from several companies for other projects, and he owns stock in several companies. PMD is a co-inventor on patents (through Duke) related to metabolomics that are unlicensed at present. Full disclosures will be made through the IJCME form. R.K.D. is an inventor on key patents in the field of metabolomics including applications for Alzheimer disease. All other authors report no disclosures.

An email with links to the Authorship Form will be sent to authors for completion after manuscripts have been submitted.

\section{Supplementary data}

Supplementary data to this article can be found online at https://doi.org/10.1016/j.jalz.2018.08.012.

\section{RESEARCH IN CONTEXT}

1. Systematic review: The authors reviewed the literature using PubMed, Google, Web of Science, and through meeting abstracts and presentations. We have cited several recent publications implicating the gut microbiome role in neuropsychiatric diseases.

2. Interpretation: This is the first study to show that serum bile acids produced in the liver and by gut microbiome are associated with cerebrospinal fluid biomarkers and brain imaging changes in Alzheimer's disease (AD). Bile acids play key role in cholesterol clearance and in maintaining energy homeostasis. Interorgan communication seems important in maintaining brain glucose metabolism, the dysregulation of which contributes to neurodegeneration in AD.

3. Future directions: Understanding gut microbiome's role in aging and related diseases opens potential new hypotheses for AD. Prospective clinical observations and validation in model systems are needed to assess causality and specific mechanisms underlying bile acid and gut microbiome dysbiosis in AD. The biochemical gut-liver-brain axis of communication should be further evaluated in AD.

\section{References}

[1] Wilkins JM, Trushina E. Application of metabolomics in Alzheimer's disease. Front Neurol 2018;8:719.

[2] Cutler RG, Kelly J, Storie K, Pedersen WA, Tammara A, Hatanpaa K, et al. Involvement of oxidative stress-induced abnormalities in ceramide and cholesterol metabolism in brain aging and Alzheimer's disease. Proc Natl Acad Sci U S A 2004;101:2070-5.

[3] Gamba P, Testa G, Sottero B, Gargiulo S, Poli G, Leonarduzzi G. The link between altered cholesterol metabolism and Alzheimer's disease. Ann N Y Acad Sci 2012;1259:54-64.

[4] Graham WV, Bonito-Oliva A, Sakmar TP. Update on Alzheimer's disease therapy and prevention strategies. Annu Rev Med 2017;68:413-30.

[5] Di Paolo G, Kim TW. Linking lipids to Alzheimer's disease: cholesterol and beyond. Nat Rev Neurosci 2011;12:284-96.

[6] Schmidt DR, Holmstrom SR, Fon Tacer K, Bookout AL, Kliewer SA, Mangelsdorf DJ. Regulation of bile acid synthesis by fat-soluble vitamins A and D. J Biol Chem 2010;285:14486-94.

[7] Klingelhoefer L, Reichmann H. Pathogenesis of Parkinson disease-the gut-brain axis and environmental factors. Nat Rev Neurol 2015; 11:625-36.

[8] Dinan TG, Cryan JF. Gut-brain axis in 2016: brain-gut-microbiota axis - mood, metabolism and behaviour. Nat Rev Gastroenterol Hepatol 2017;14:69-70.

[9] Dinan TG, Cryan JF. Gut instincts: microbiota as a key regulator of brain development, ageing and neurodegeneration. J Physiol 2017; 595:489-503.

[10] Kueider AM, Tanaka T, An Y, Kitner-Triolo MH, Palchamy E, Ferrucci L, et al. State- and trait-dependent associations of vitaminD with brain function during aging. Neurobiol Aging 2016;39:38-45. 
[11] Littlejohns TJ, Henley WE, Lang IA, Annweiler C, Beauchet O, Chaves PHM, et al. Vitamin D and the risk of dementia and Alzheimer disease. Neurology 2014;83:920-8.

[12] Quinn M, DeMorrow S. Bile in the brain? A role for bile acids in the central nervous system. J Cell Sci Ther 2012;3.

[13] Mertens KL, Kalsbeek A, Soeters MR, Eggink HM. Bile acid signaling pathways from the enterohepatic circulation to the central nervous system. Front Neurosci 2017;11:617.

[14] Xie G, Zhang S, Zheng X, Jia W. Metabolomics approaches for characterizing metabolic interactions between host and its commensal microbes. Electrophoresis 2013;34:2787-98.

[15] Luan H, Wang X, Cai Z. Mass spectrometry-based metabolomics: Targeting the crosstalk between gut microbiota and brain in neurodegenerative disorders. Mass Spec Rev 2017:1-12. https://doi.org/10.1002/ mas. 21553.

[16] Ackerman HD, Gerhard GS. Bile acids in neurodegenerative disorders. Front Aging Neurosci 2016;8.

[17] Gacias M, Gaspari S, Santos PM, Tamburini S, Andrade M, Zhang F, et al. Microbiota-driven transcriptional changes in prefrontal cortex override genetic differences in social behavior. eLife 2016;5.

[18] Cryan JF, Dinan TG. Mind-altering microorganisms: the impact of the gut microbiota on brain and behaviour. Nat Rev Neurosci 2012; 13:701-12.

[19] Littman DR, Pamer EG. Role of the commensal microbiota in normal and pathogenic host immune responses. Cell host \& microbe 2011; $10: 311-23$.

[20] Serres S, Anthony DC, Jiang Y, Broom KA, Campbell SJ, Tyler DJ, et al. Systemic inflammatory response reactivates immune-mediated lesions in rat brain. J Neurosci 2009;29:4820-8.

[21] Erny D, Hrabe de Angelis AL, Jaitin D, Wieghofer P, Staszewski O, David E, et al. Host microbiota constantly control maturation and function of microglia in the CNS. Nat Neurosci 2015;18:965-77.

[22] Erny D, Hrabe de Angelis AL, Prinz M. Communicating systems in the body: how microbiota and microglia cooperate. Immunology 2017; 150:7-15.

[23] Foster JA, McVey Neufeld KA. Gut-brain axis: how the microbiome influences anxiety and depression. Trends Neurosciences 2013; 36:305-12.

[24] Maes M, Kubera M, Leunis JC. The gut-brain barrier in major depression: intestinal mucosal dysfunction with an increased translocation of LPS from gram negative enterobacteria (leaky gut) plays a role in the inflammatory pathophysiology of depression. Neuro Endocrinol Lett 2008;29:117-24.

[25] Ogbonnaya ES, Clarke G, Shanahan F, Dinan TG, Cryan JF, O'Leary OF. Adult hippocampal neurogenesis is regulated by the microbiome. Biol Psychiatry 2015;78:e7-9.

[26] Berthoud H-R, Kressel M, Raybould HE, Neuhuber WL. Vagal sensors in the rat duodenal mucosa: distribution and structure as revealed by in vivo DiI-tracing. Anat Embryol 1995;191:203-12.

[27] Greenberg N, Grassano A, Thambisetty M, Lovestone S, LegidoQuigley C. A proposed metabolic strategy for monitoring disease progression in Alzheimer's disease. Electrophoresis 2009;30:1235-9.

[28] Harach T, Marungruang N, Duthilleul N, Cheatham V, Mc Coy KD, Frisoni G, et al. Reduction of Abeta amyloid pathology in APPPS1 transgenic mice in the absence of gut microbiota. Scientific Rep 2017;7:41802.

[29] Jack CR Jr, Bennett DA, Blennow K, Carrillo MC, Feldman HH, Frisoni GB, et al. A/T/N: An unbiased descriptive classification scheme for Alzheimer disease biomarkers. Neurology 2016; 87:539-47.

[30] Jack CR Jr, Bennett DA, Blennow K, Carrillo MC, Dunn B, Budd Haeberlein S, et al. NIA-AA Research Framework: Towards a Biological Definition of Alzheimer's Disease. Alzheimer's Association International Conference 2017. London, England: Alzheimer's Association; 2017. p. 1-57.

[31] Jack CR Jr, Bennett DA, Blennow K, Carrillo MC, Dunn B, Haeberlein SB, et al. NIA-AA research framework: toward a biolog- ical definition of Alzheimer's disease. Alzheimers Dement 2018; 14:535-62.

[32] Saykin AJ, Shen L, Yao X, Kim S, Nho K, Risacher SL, et al. Genetic studies of quantitative $\mathrm{MCI}$ and $\mathrm{AD}$ phenotypes in ADNI: Progress, opportunities, and plans. Alzheimers Dement 2015; 11:792-814.

[33] Weiner MW, Veitch DP, Aisen PS, Beckett LA, Cairns NJ, Green RC, et al. Recent publications from the Alzheimer's disease neuroimaging initiative: reviewing progress toward improved AD clinical trials. Alzheimers Dement 2017;13:e1-85.

[34] Pham HT, Arnhard K, Asad YJ, Deng L, Felder TK, St. JohnWilliams L, et al. Inter-laboratory robustness of next-generation bile acid study in mice and humans: International Ring Trial involving 12 laboratories. J Appl Lab Med 2016;3.

[35] Toledo JB, Arnold M, Kastenmüller G, Chang R, Baillie RA, Han X, et al. Metabolic network failures in Alzheimer's disease: a biochemical road map. Alzheimers Dement 2017;13:965-84.

[36] Jack CR Jr, Bernstein MA, Borowski BJ, Gunter JL, Fox NC, Thompson PM, et al. Update on the magnetic resonance imaging core of the Alzheimer's disease neuroimaging initiative. Alzheimers Dement 2010;6:212-20.

[37] Jack CR Jr, Bernstein MA, Fox NC, Thompson P, Alexander G, Harvey D, et al. The Alzheimer's Disease Neuroimaging Initiative (ADNI): MRI methods. J Magn Reson Imaging 2008;27:685-91.

[38] Kim S, Swaminathan S, Inlow M, Risacher SL, Nho K, Shen L, et al. Influence of genetic variation on plasma protein levels in older adults using a multi-analyte panel. PLoS One 2013;8:e70269.

[39] Nho K, Corneveaux JJ, Kim S, Lin H, Risacher SL, Shen L, et al. Whole-exome sequencing and imaging genetics identify functional variants for rate of change in hippocampal volume in mild cognitive impairment. Mol Psychiatry 2013;18:781-7.

[40] Nho K, Kim S, Risacher SL, Shen L, Corneveaux JJ, Swaminathan S, et al. Protective variant for hippocampal atrophy identified by whole exome sequencing. Ann Neurol 2015;77:547-52.

[41] Fischl B, Sereno MI, Dale AM. Cortical surface-based analysis. II: inflation, flattening, and a surface-based coordinate system. Neuroimage 1999;9:195-207.

[42] Dale AM, Fischl B, Sereno MI. Cortical surface-based analysis. I. Segmentation and surface reconstruction. Neuroimage 1999; 9:179-94.

[43] Chung MK, Worsley KJ, Nacewicz BM, Dalton KM, Davidson RJ. General multivariate linear modeling of surface shapes using SurfStat. NeuroImage 2010;53:491-505.

[44] Risacher SL, Kim S, Nho K, Foroud T, Shen L, Petersen RC, et al. APOE effect on Alzheimer's disease biomarkers in older adults with significant memory concern. Alzheimers Dement 2015;11:1417-29.

[45] Bittner T, Zetterberg H, Teunissen CE, Ostlund RE Jr, Militello M, Andreasson U, et al. Technical performance of a novel, fully automated electrochemiluminescence immunoassay for the quantitation of beta-amyloid (1-42) in human cerebrospinal fluid. Alzheimers Dement 2016;12:517-26.

[46] Hansson O, Seibyl J, Stomrud E, Zetterberg H, Trojanowski JQ, Bittner T, et al. CSF biomarkers of Alzheimer's disease concord with amyloid-beta PET and predict clinical progression: a study of fully automated immunoassays in BioFINDER and ADNI cohorts. Alzheimers Dement 2018; https://doi.org/10.1016/j.jalz.2018.01.010.

[47] Benjamini Y, Hochberg Y. Controlling the false discovery rate - a practical and powerful approach to multiple testing. J Roy Stat Soc B Met 1995;57:289-300.

[48] Hagler DJ Jr, Saygin AP, Sereno MI. Smoothing and cluster thresholding for cortical surface-based group analysis of fMRI data. Neuroimage 2006;33:1093-103.

[49] Hayasaka S, Phan KL, Liberzon I, Worsley KJ, Nichols TE. Nonstationary cluster-size inference with random field and permutation methods. Neuroimage 2004;22:676-87.

[50] Worsley KJ, Taylor JE, Tomaiuolo F, Lerch J. Unified univariate and multivariate random field theory. Neuroimage 2004;23:S189-95. 
[51] Olsson B, Lautner R, Andreasson U, Ohrfelt A, Portelius E, Bjerke M, et al. CSF and blood biomarkers for the diagnosis of Alzheimer's disease: a systematic review and meta-analysis. Lancet Neurol 2016;15:673-84.

[52] Blennow K, Hampel H, Weiner M, Zetterberg H. Cerebrospinal fluid and plasma biomarkers in Alzheimer disease. Nat Rev Neurol 2010;6:131-44.

[53] Herukka SK, Simonsen AH, Andreasen N, Baldeiras I, Bjerke M, Blennow K, et al. Recommendations for cerebrospinal fluid Alzheimer's disease biomarkers in the diagnostic evaluation of mild cognitive impairment. Alzheimers Dement 2017;13:285-95.

[54] Handels RLH, Vos SJB, Kramberger MG, Jelic V, Blennow K, van Buchem M, et al. Predicting progression to dementia in persons with mild cognitive impairment using cerebrospinal fluid markers. Alzheimers Dement 2017;13:903-12.

[55] Dean RA, Shaw LM. Use of cerebrospinal fluid biomarkers for diagnosis of incipient Alzheimer disease in patients with mild cognitive impairment. Clin Chem 2010;56:7-9.

[56] Shaw LM, Vanderstichele H, Knapik-Czajka M, Clark CM, Aisen PS, Petersen RC, et al. Cerebrospinal fluid biomarker signature in Alzheimer's disease neuroimaging initiative subjects. Ann Neurol 2009; 65:403-13.

[57] Risacher SL, Saykin AJ. Neuroimaging biomarkers of neurodegenerative diseases and dementia. Semin Neurol 2013;33:386-416.

[58] Risacher SL, Shen L, West JD, Kim S, McDonald BC, Beckett LA, et al. Longitudinal MRI atrophy biomarkers: relationship to conversion in the ADNI cohort. Neurobiol Aging 2010;31:1401-18.

[59] Risacher SL, Saykin AJ, West JD, Shen L, Firpi HA, McDonald BC, et al. Baseline MRI predictors of conversion from MCI to probable AD in the ADNI cohort. Curr Alzheimer Res 2009;6:347-61.

[60] Ishii K, Willoch F, Minoshima S, Drzezga A, Ficaro EP, Cross DJ, et al. Statistical brain mapping of 18F-FDG PET in Alzheimer's disease: validation of anatomic standardization for atrophied brains. J Nucl Med 2001;42:548-57.

[61] Marcus C, Mena E, Subramaniam RM. Brain PET in the diagnosis of Alzheimer's disease. Clin Nucl Med 2014;39:e413-22. quiz e23-6.

[62] Ewers M, Insel PS, Stern Y, Weiner MW. Alzheimer's disease neuroimaging I. Cognitive reserve associated with FDG-PET in preclinical Alzheimer disease. Neurology 2013;80:1194-201.

[63] Daulatzai MA. Chronic functional bowel syndrome enhances gutbrain axis dysfunction, neuroinflammation, cognitive impairment, and vulnerability to dementia. Neurochem Res 2014;39:624-44.

[64] Daulatzai MA. Role of stress, depression, and aging in cognitive decline and Alzheimer's disease. Curr Top Behav neurosciences 2014;18:265-96.

[65] Berger AL. Insulin resistance and reduced brain glucose metabolism in the aetiology of Alzheimer's disease. J Insulin Resist 2016;1(No 1).

[66] Naseer MI, Bibi F, Alqahtani MH, Chaudhary AG, Azhar EI, Kamal MA, et al. Role of gut microbiota in obesity, type 2 diabetes and Alzheimer's disease. CNS Neurol Disord Drug Targets 2014;13:305-11.

[67] Alam MZ, Alam Q, Kamal MA, Abuzenadah AM, Haque A. A possible link of gut microbiota alteration in type 2 diabetes and Alzheimer's disease pathogenicity: an update. CNS Neurol Disord Drug Targets 2014;13:383-90.

[68] Zhao Y, Lukiw WJ. Microbiome-generated amyloid and potential impact on amyloidogenesis in Alzheimer's disease (AD). J Nat Sci $2015 ; 1$

[69] Islam KB, Fukiya S, Hagio M, Fujii N, Ishizuka S, Ooka T, et al. Bile acid is a host factor that regulates the composition of the cecal microbiota in rats. Gastroenterology 2011;141:1773-81.

[70] Vogt NM, Kerby RL, Dill-McFarland KA, Harding SJ, Merluzzi AP, Johnson SC, et al. Gut microbiome alterations in Alzheimer's disease. Scientific Rep 2017;7:13537.

[71] Cattaneo A, Cattane N, Galluzzi S, Provasi S, Lopizzo N, Festari C, et al. Association of brain amyloidosis with pro-inflammatory gut bacterial taxa and peripheral inflammation markers in cognitively impaired elderly. Neurobiol Aging 2017;49:60-8.
[72] Shivaram KN, Winklhofer-Roob BM, Straka MS, Devereaux MW, Everson G, Mierau GW, et al. The effect of idebenone, a coenzyme $\mathrm{Q}$ analogue, on hydrophobic bile acid toxicity to isolated rat hepatocytes and hepatic mitochondria. Free Radic Biol Med 1998;25:480-92.

[73] Sokol RJ, McKim JM Jr, Goff MC, Ruyle SZ, Devereaux MW, Han D, et al. Vitamin E reduces oxidant injury to mitochondria and the hepatotoxicity of taurochenodeoxycholic acid in the rat. Gastroenterology 1998;114:164-74.

[74] Sokol RJ, Winklhofer-Roob BM, Devereaux MW, McKim JM Jr. Generation of hydroperoxides in isolated rat hepatocytes and hepatic mitochondria exposed to hydrophobic bile acids. Gastroenterology 1995; 109:1249-56.

[75] Rodrigues CM, Fan G, Ma X, Kren BT, Steer CJ. A novel role for ursodeoxycholic acid in inhibiting apoptosis by modulating mitochondrial membrane perturbation. J Clin Invest 1998;101:2790-9.

[76] Patel T, Gores GJ. Inhibition of bile-salt-induced hepatocyte apoptosis by the antioxidant lazaroid U83836E. Toxicol Appl Pharmacol 1997; $142: 116-22$.

[77] Leloup C, Magnan C, Benani A, Bonnet E, Alquier T, Offer G, et al. Mitochondrial reactive oxygen species are required for hypothalamic glucose sensing. Diabetes 2006;55:2084-90.

[78] Fioramonti X, Chretien C, Leloup C, Penicaud L. Recent advances in the cellular and molecular mechanisms of hypothalamic neuronal glucose detection. Front Physiol 2017;8:875.

[79] Santos RX, Correia SC, Wang X, Perry G, Smith MA, Moreira PI, et al. Alzheimer's disease: diverse aspects of mitochondrial malfunctioning. Int J Clin Exp Pathol 2010;3:570-81.

[80] Moreira PI, Carvalho C, Zhu X, Smith MA, Perry G. Mitochondrial dysfunction is a trigger of Alzheimer's disease pathophysiology. Biochim Biophys Acta 2010;1802:2-10.

[81] Markesbery WR. The role of oxidative stress in Alzheimer disease. Arch Neurol 1999;56:1449-52.

[82] Scholmerich J, Becher MS, Schmidt K, Schubert R, Kremer B, Feldhaus $\mathrm{S}$, et al. Influence of hydroxylation and conjugation of bile salts on their membrane-damaging properties-studies on isolated hepatocytes and lipid membrane vesicles. Hepatology 1984;4:661-6.

[83] Rodrigues CM, Ma X, Linehan-Stieers C, Fan G, Kren BT, Steer CJ. Ursodeoxycholic acid prevents cytochrome $\mathrm{c}$ release in apoptosis by inhibiting mitochondrial membrane depolarization and channel formation. Cell Death Differ 1999;6:842-54.

[84] Rodrigues CM, Stieers CL, Keene CD, Ma X, Kren BT, Low WC, et al. Tauroursodeoxycholic acid partially prevents apoptosis induced by 3-nitropropionic acid: evidence for a mitochondrial pathway independent of the permeability transition. J Neurochem 2000;75:2368-79.

[85] Rolo AP, Oliveira PJ, Moreno AJ, Palmeira CM. Bile acids affect liver mitochondrial bioenergetics: possible relevance for cholestasis therapy. Toxicol Sci 2000;57:177-85.

[86] Rolo AP, Palmeira CM, Holy JM, Wallace KB. Role of Mitochondrial Dysfunction in Combined Bile Acid-Induced Cytotoxicity: The Switch Between Apoptosis and Necrosis. Toxicol Sci 2004; 79:196-204.

[87] Rolo AP, Palmeira CM, Wallace KB. Mitochondrially mediated synergistic cell killing by bile acids. Biochim Biophys Acta 2003; 1637:127-32.

[88] Krähenbühl S, Fischer S, Talos C, Reichen PJ. Ursodeoxycholate protects oxidative mitochondrial metabolism from bile acid toxicity: Dose-response study in isolated rat liver mitochondria. Hepatology 1994;20:1595-601.

[89] Li M, Wang B, Zhang M, Rantalainen M, Wang S, Zhou H, et al. Symbiotic gut microbes modulate human metabolic phenotypes. Proc Natl Acad Sci 2008;105:2117.

[90] Weir TL, Manter DK, Sheflin AM, Barnett BA, Heuberger AL, Ryan EP. Stool Microbiome and Metabolome Differences between Colorectal Cancer Patients and Healthy Adults. PLos One 2013; 8:e70803. 